\title{
Optical time-slot swapping based on parametric wavelength exchange
}

Xing Xu, Mengzhe Shen, T. I. Yuk, K. K. Y. Wong

Xing Xu, Mengzhe Shen, T. I. Yuk, K. K. Y. Wong, "Optical time-slot swapping based on parametric wavelength exchange," Proc. SPIE 7630, Passive Components and Fiber-based Devices VI, 763002 (1 December 2009); doi: $10.1117 / 12.852044$

SPIE Event: Asia Communications and Photonics, 2009, Shanghai, Shanghai , China 


\title{
Optical Time-Slot Swapping Based on Parametric Wavelength Exchange
}

\author{
Xing Xu ${ }^{a}$, Mengzhe Shen ${ }^{b}$, T. I. Yuk ${ }^{a}$ and Kenneth K. Y. Wong ${ }^{* a}$ \\ ${ }^{a}$ Photonic Systems Lab, Department of Electrical and Electronic Engineering, \\ The University of Hong Kong, Pokfulam Road, Hong Kong; \\ ${ }^{b}$ Department of Electrical and Computer Engineering, University of British Columbia, Vancouver \\ V6T 1Z4, Canada
}

\begin{abstract}
We have experimentally demonstrated simultaneous $10-\mathrm{Gb} / \mathrm{s}$ optical time-slot swapping between return-to-zero (RZ) signal format and non-return-to-zero (NRZ) signal format based on the parametric wavelength exchange (PWE) in the highly-nonlinear dispersion shifted fiber (HNL-DSF). Original RZ and NRZ signals located at two different wavelengths are exchanged after the PWE process. After exchanging between RZ and NRZ signals, the swapping ability involving two different signal formats within particular time slot has been proved. Clear open eye diagrams of periodic mixed RZ and NRZ signals are recorded on both wavelengths.
\end{abstract}

Keywords: Four Wave Mixing (FWM), Optical Signal Processing, Wavelength Conversion, Wavelength Exchange (WE), Time-slot Swapping, Optical Packet Switch

\section{INTRODUCTION}

Parametric wavelength exchange (PWE), which realizes the simultaneous wavelength conversion between two signals located at two different wavelengths, relies on parametric effects in the highly-nonlinear dispersion shifted fibers (HNLDSF) [1,2]. In recent research [3], PWE has been comprehensively studied both theoretically and experimentally.

Lately, optical time-slot interchange with fiber-based wavelength conversion and tunable delays [4] and optical label swapping for the network application [5] have attracted significant interests, and either FWM-based wavelength conversion scheme [4] or SOA-based wavelength converter approach [5] was studied comprehensively. However, in these experimental demonstrations, only one wavelength conversion for individual signal can be fulfilled within the process. For those applications when simultaneous exchange between two signals is needed, these techniques are incapable of help. However, this problem can be easily solved by utilizing PWE. The benefits of wavelength exchange are obvious, such as two wavelength conversion could be done in one process instead of complex setup and this will surely reduce the complexity of the whole signal processing system in use. In this paper, we propose and demonstrate not only concurrent wavelength conversion between two signals but involving two different data format in the same process. Based on the scheme of pulsed pump parametric wavelength exchange (PWE) in the highly-nonlinear dispersion shifted fiber (HNL-DSF), an RZ-NRZ optical signal time-slot swapper is successfully fulfilled. Besides the advantage of synchronous wavelength conversion, parametric wavelength exchange (PWE) has several unique merits, such as simultaneous power exchange between different wavelengths [3], low-noise operation [6], and polarization independence [7], which have make it a perfect choice for optical packet switch, optical add-drop and etc. Comparing to the previous research on packet-switch application by parametric wavelength exchange (PWE) [8], in order to make the whole process more reasonable in our experiment we employ a practical pseudorandom binary sequence (PRBS) signal with the total length of $2^{7}-1$ bits instead of preset data string adopted in [8] which result in a partly incomplete eye diagram at the system output. Meanwhile, for the reason of emphasizing the contrast in the output mixed signal eye-diagram, both NRZ

*kywong@eee.hku.hk; phone 85228578483

Passive Components and Fiber-based Devices VI, edited by Perry P. Shum,

Proc. of SPIE-OSA-IEEE Asia Communications and Photonics, SPIE Vol. 7630

763002 - @ 2009 SPIE-OSA-IEEE · CCC code: 0277-786X/09/\$18 - doi: 10.1117/12.852044

Proc. of SPIE-OSA-IEEE/ Vol. $7630763002-1$ 
format and RZ format signals are applied in our experiment. The output signal eye diagrams are utilized to analyze the performance of our time-slot swapper.

\section{PRINCIPLE AND EXPERIMENTAL SETUP}

\subsection{Principle}

Due to its unique unit exchange characteristics, parametric wavelength exchange (PWE) has been proposed to operate in the wavelength domain for optical signal stream. with proper wavelength allocations, one signal wavelength at $\lambda_{s}$ and another idler wavelength at $\lambda_{i}$, while co-propagating with two strong pumps wavelength at $\lambda_{p 1}$ and $\lambda_{p 2}$ respectively in the highly-nonlinear dispersion shifted fiber (HNL-DSF), will periodically exchange their power according to the function of the fiber length.

To explain the process of parametric wavelength exchange more theoretically, four waves including signal, idler and two pumps are arranged symmetrically with respect to the zero dispersion wavelength $\lambda_{0}$ of the Highly Nonlinear Dispersion Shifted Fiber (HNL-DSF), such that the frequencies of pumps and signals $\omega_{j}(j=1,2,3,4)$ satisfy the following equation:

$$
\omega_{1}+\omega_{4}=\omega_{2}+\omega_{3}=2 \omega_{0} .
$$

Where $\omega_{1}, \omega_{2}$ are the frequencies of pumps and $\omega_{3}, \omega_{4}$ are the frequencies of the signal and the idler. $\omega_{0}$ is the frequency corresponding to the zero dispersion wavelength $\lambda_{0}$. Based on the above equation, we could also rearrange the pumps or signals such that, both pumps are located in normal dispersion region of the fiber (Type I), both pumps are located in anomalous dispersion region of the fiber (Type II), or with each located in the normal and anomalous dispersion region of the fiber (Type III). Except the wavelength allocation, the state of polarization (SOP) of both signals and pumps could also affect the performance of PWE. Different types of PWE are shown in the following Fig. 1:
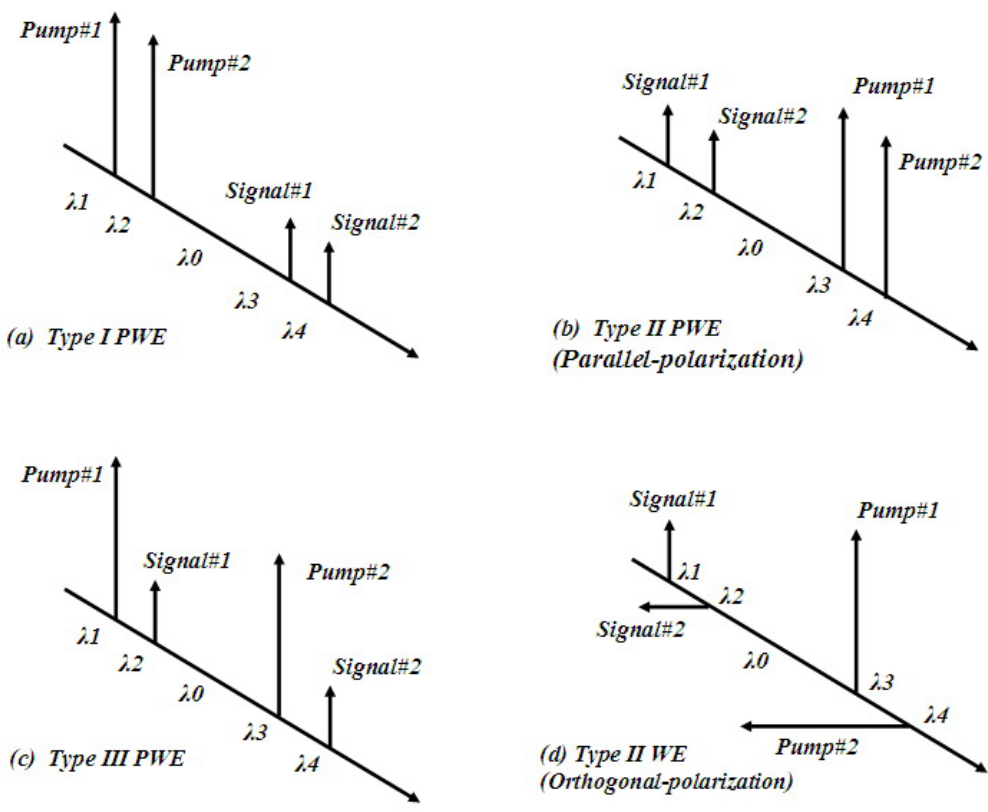

Figure 1. Wavelength allocations for different types of parametric wavelength exchange (PWE)

Additionally, although both parallel-polarized and orthogonal-polarized PWE (Fig. 1 (b) and Fig. 1 (d)) are shown above, in the practical experiments, orthogonal-polarization scheme between pumps and signals are preferred due to its 
superior characteristic of suppressing other undesired four wave mixing (FWM) components generated simultaneously during the PWE process, and these FWM components could seriously degrade the quality of both the signal and idler [3]. In this paper, we utilize type II PWE with parallel polarization in our experiment.

\subsection{Experimental Setup}

In order to achieve synchronous interchange amid two distinct signal formats, strictly aligned pumps with certain length of swapping window must be used. Fig.2 shows the experimental setup of the proposed Time-slot Swapper based on PWE in the HNL-DSF. The RZ signal wavelength at $1534 \mathrm{~nm}$ is generated by intensity-modulating a pulse train with the repetition rate of $10 \mathrm{GHz}$ sourced from a low power mode-locked laser diode (MLLD). Due to the low output power of the MLLD, it is first amplified by an erbium-doped fiber amplifier (EDFA4) before passing through the polarization controller (PC7) to minimize the insertion loss of the subsequent polarization sensitive Mach-Zehnder modulator (MZM1). To achieve clear comparison, one $10 \mathrm{~Gb} / \mathrm{s} \mathrm{NRZ} \mathrm{signal} \mathrm{is} \mathrm{then} \mathrm{generated} \mathrm{from} \mathrm{a} \mathrm{continuous} \mathrm{wave} \mathrm{(CW)} \mathrm{tunable}$ laser source (TLS3) wavelength at $1529 \mathrm{~nm}$, and intensity-modulated by MZM2. Before combining with the RZ signal branch, an erbium-doped fiber amplifier (EDFA3) is used to amplify the NRZ signal to an average power of $15 \mathrm{dBm}$. The PRBS length used for both the RZ and NRZ signal is $2^{7}-1$ bits.

Besides two signals, double CW pumps, located at $1549 \mathrm{~nm}$ (TLS1) and $1554 \mathrm{~nm}$ (TLS2), respectively, are firstly intensity-modulated by MZM3 with a periodical quasi-square-pulse gating signal source, which is the trigger pattern with the repetition frequency of $624 \mathrm{MHz}$ and $50 \%$ duty ratio from the pattern generator of a $10 \mathrm{~Gb} / \mathrm{s}$ BERT system. To suppress stimulated Brillouin scattering (SBS), two pumps are phase dithered with a phase modulator (PM) driven by a $10 \mathrm{~Gb} / \mathrm{s}$ PRBS with the length of $2^{23}-1$, they are pre-amplified by EDFA1 before boosted by EDFA2 to an average output power of $26 \mathrm{dBm}$. Polarization controllers (PC1, PC2 and PC3) are inserted before AM1 and PM to reduce the insertion loss. Two optical band-pass filters (OBPF1 and OBPF2) are inserted after WDM coupler (WDMC1) in order to reduce the amplified spontaneous emission (ASE) noise from EDFA1. The alignment of both pulsed pumps is carried out by optical delay line (ODL2). Meanwhile, ODL1 is used to align pumps with signals. The state of polarization (SOP) of pumps and signals are controlled by polarization controllers (PC4, PC5, PC8, and PC9) at each branch to satisfy the orthogonal polarization condition before they are combined and launched into a $1 \mathrm{~km}$ HNL-DSF for PWE. The nonlinear coefficient $\gamma$, the zero-dispersion wavelength $\lambda_{0}$ and the dispersion slope $d D / d \lambda$ of HNL-DSF are $12 \mathrm{~W}^{-1} \mathrm{~km}^{-1}, 1541 \mathrm{~nm}$ and $0.03 \mathrm{ps} / \mathrm{nm}^{2} \mathrm{~km}$, respectively.

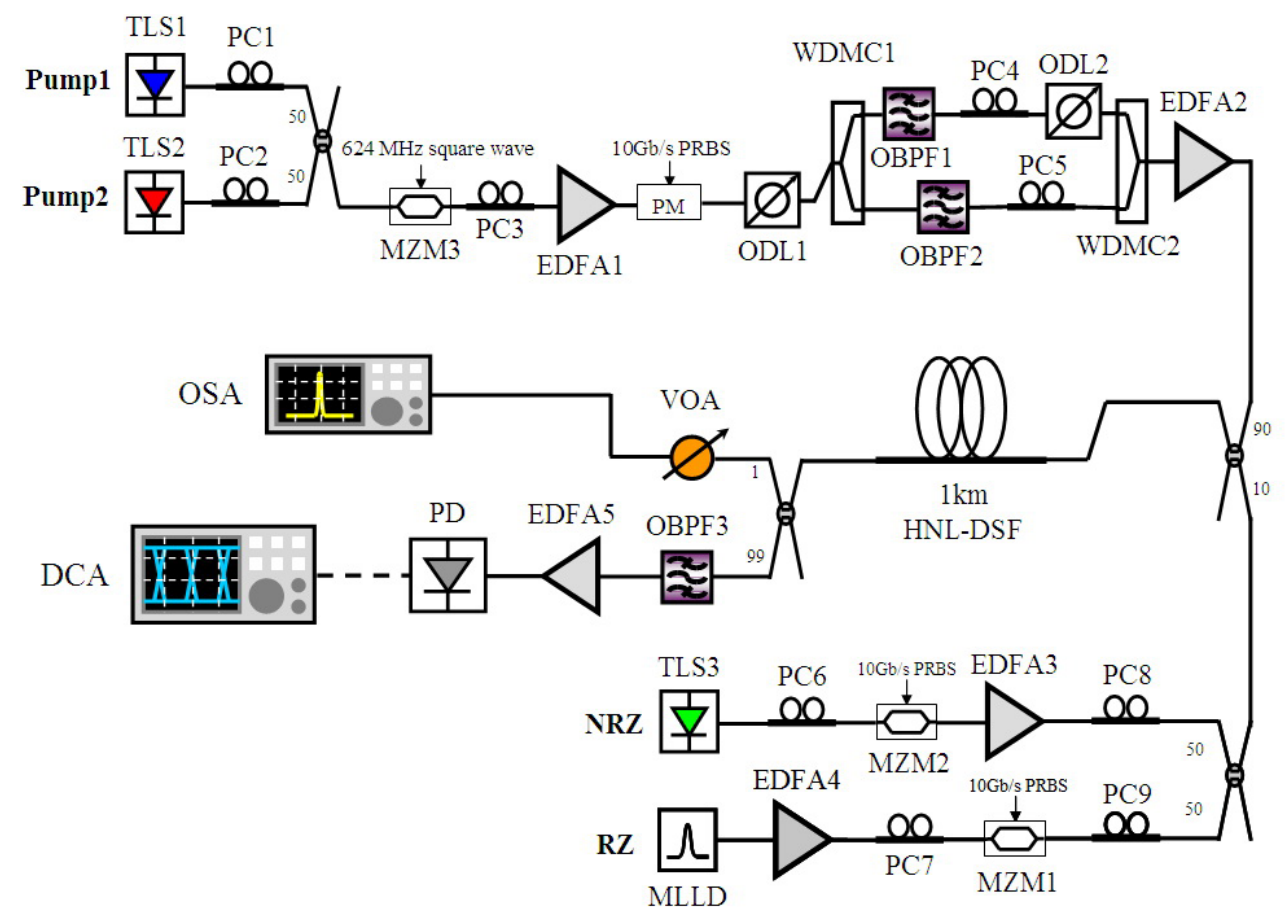

Figure 2. Experimental setup of the RZ-NRZ time-slot swapping based on PWE 
Fig. 3 shows the waveform for the quasi-square-pulse stream used in the experiment as the gating signal for two pumps. Eye diagrams for the original RZ and NRZ signals before the fiber input are shown in Fig. 4 and Fig. 5, while in Fig. 6 the eye diagram of the RZ signal collected from optical sampling oscilloscope (OSO) is shown to illustrate the real width of RZ signal pulse. The optical spectrums before the input and after the output of the highly nonlinear dispersion shifted fiber (HNL-DSF) are shown together in Fig. 7. After the time-slot swapping process, the exchanged signals are filtered out separately by OBPF3 and then amplified by EDFA5 before being sent to the digital communication analyzer (DCA) for eye diagram analysis.

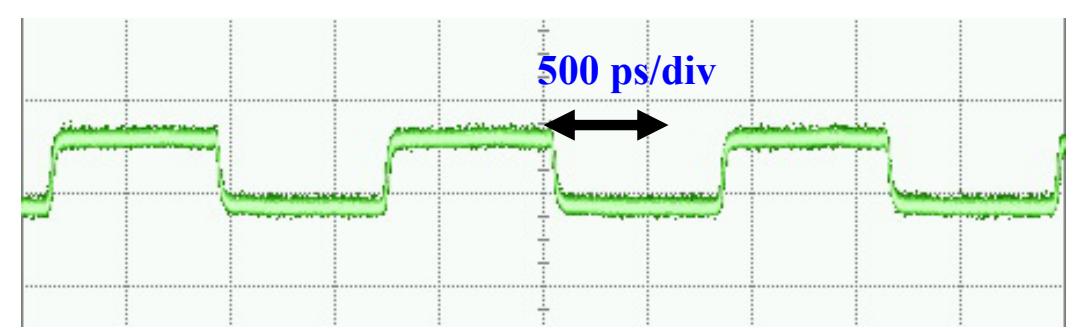

Figure 3. Square wave used in the experiment as the gating signal for the pumps

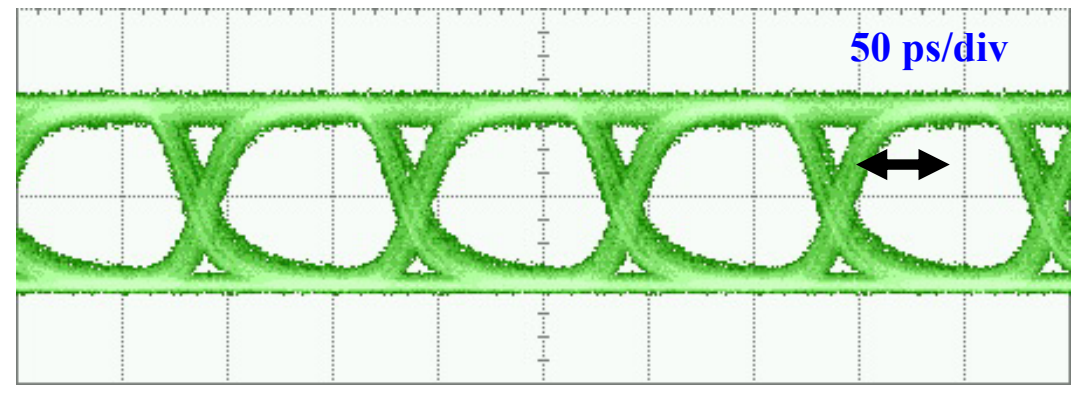

Figure 4. Original NRZ signal eye diagrams from DCA

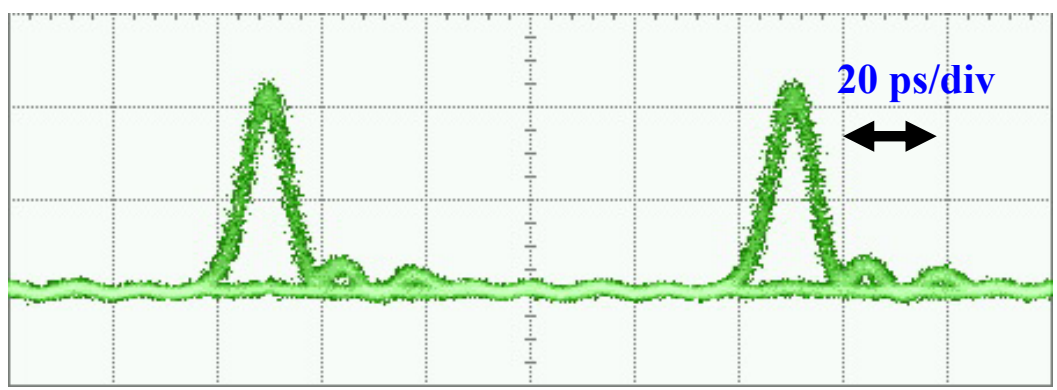

Figure 5. Original RZ signal eye diagrams from DCA

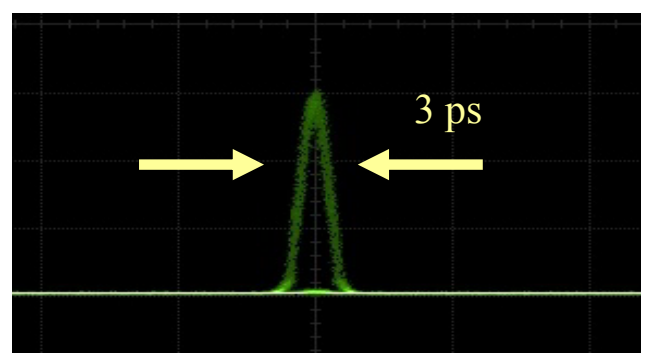

Figure 6. Original RZ signal eye diagram displayed by OSO 


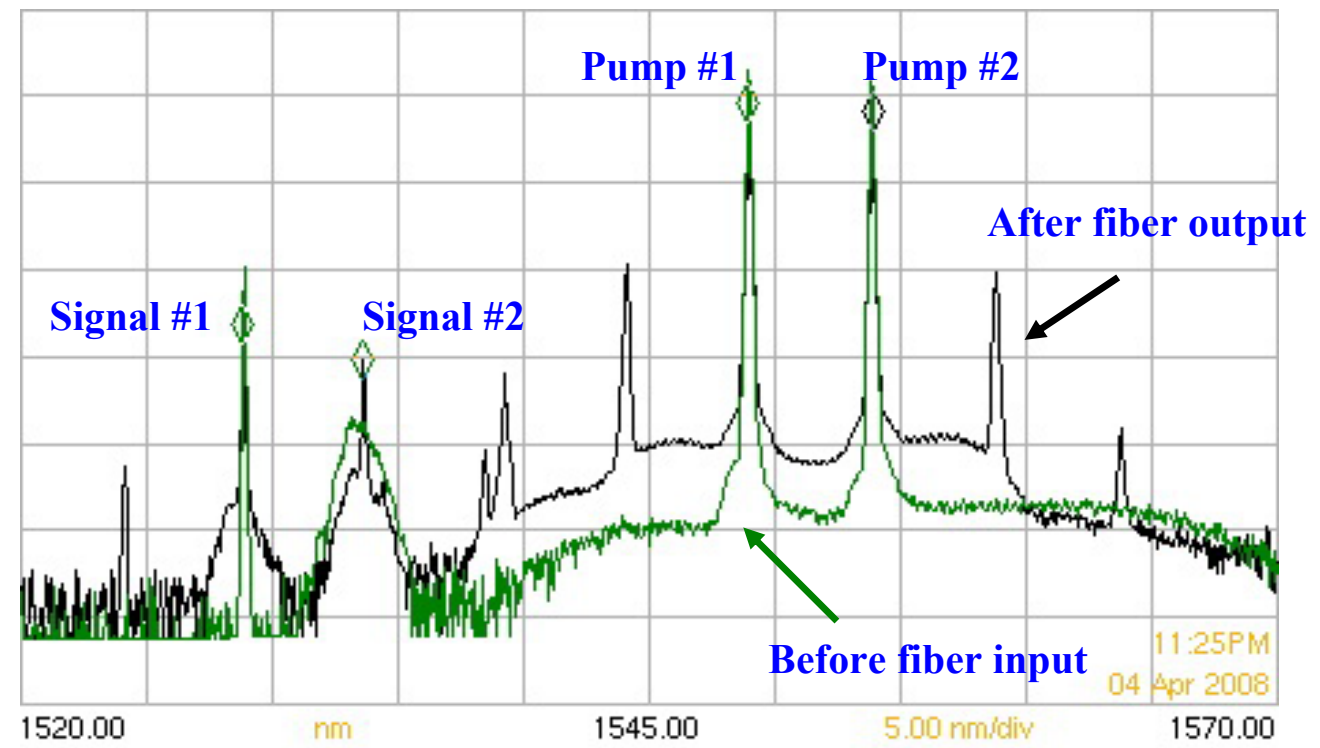

Figure 7. Spectrums before the input and after the output of the Highly-Nonlinear Dispersion Shifted Fiber (HNL-DSF)

\section{RESULTS AND DISCUSSION}

Fig. 8 lists the mixed RZ and NRZ eye diagrams for both wavelengths in details. The signals inside the timeslot, as shown in dashed boxes of Fig. 8 (a) and (b), are chosen to be swapped during the process of PWE. From the graphs we could find that successful exchange of chosen signal strings has been fulfilled. Clear and widely opening eye diagrams are observed for both wavelengths (Fig. 9 and Fig. 10). The obvious ripples observed at the end of each RZ eye diagram are due to the limited operating bandwidth of a $53 \mathrm{GHz}$ photo-detector (PD) using in our experiment. Additionally, the noise introduced during the PWE is inherited from the poor signal-to-noise ratio (SNR) of the two pumps after the high power erbium doped fiber laser (EDFA2) and it can be improved if the ASE noise of pumps can be reduced by introducing a narrow bandwidth and high suppression level fiber Bragg grating into the setup[2].

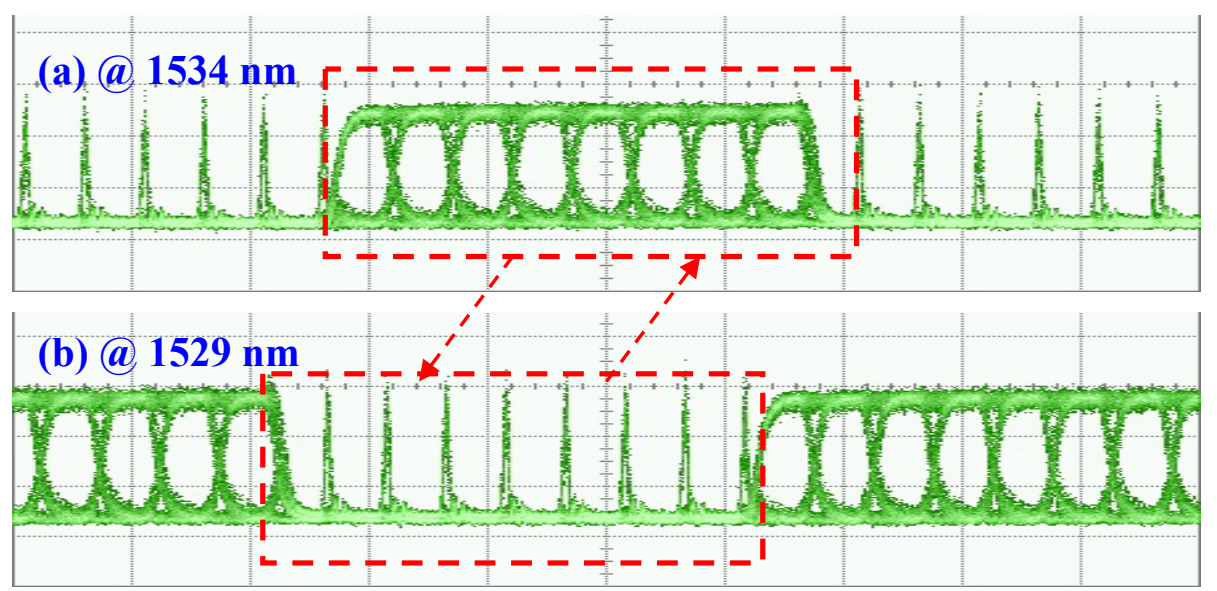

Figure 8. Output RZ-NRZ signal mixed eye diagrams 

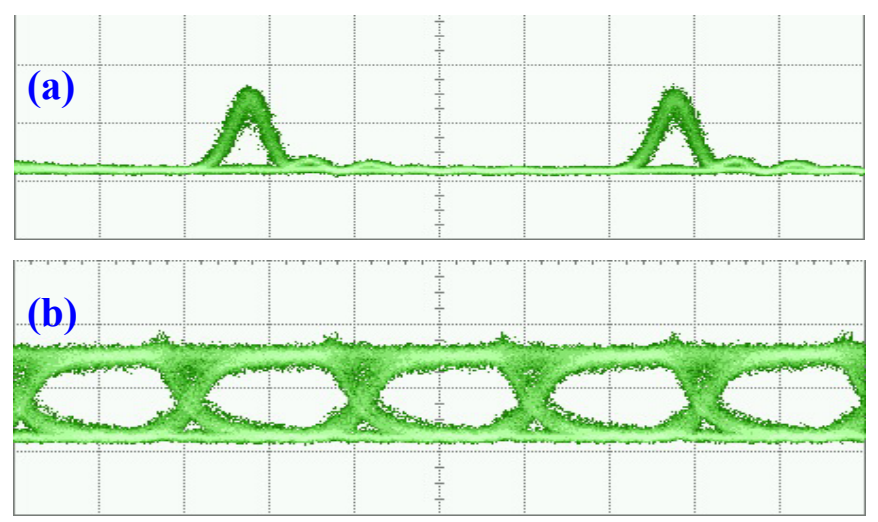

Figure 9. RZ and NRZ signals in details at the wavelength of $1534 \mathrm{~nm}$ : (a) Original residual RZ signal; (b) Swapped in NRZ signal

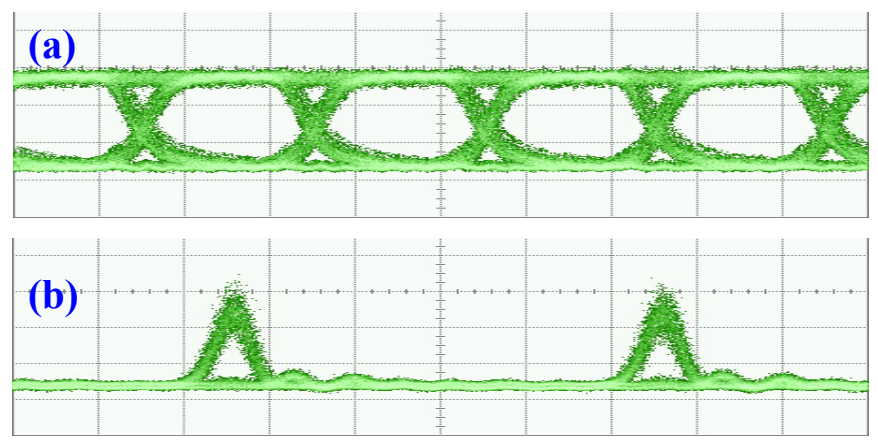

Figure 10. NRZ and RZ signals in details at the wavelength of $1529 \mathrm{~nm}$ : (a) Original residual NRZ signal; (b) Swapped in RZ signal

\section{CONCLUSIONS}

We have successfully demonstrated an optical time-slot swapper based on parametric wavelength exchange (PWE) in the highly-nonlinear dispersion shifted fiber (HNL-DSF). Simultaneous time-slot swapping of one NRZ signal with another $\mathrm{RZ}$ signal is achieved during the experiment. Clear and widely open eye diagrams have been obtained for both individual RZ, NRZ signals and mixed ones.

\section{ACKNOWLEDGEMENT}

The work described in this paper was partially supported by grants from the research Grants Council of the Hong Kong Special Administrative Region, China (Project No. HKU 7172/07E and HKU 7179/08E). The authors would also like to acknowledge Alnair Labs for the OSO, and Sumitomo Electric Industries for the HNL-DSF.

\section{REFERENCES}

[1]. M. E. Marhic, et.al., "Widely Tunable Spectrum Translation and Wavelength Exchange by FWM in Optical Fibers" Opt. Let. 21, 1906-1908 (1996). 
[2]. K. Y. Wong, et.al., "Demonstration of Wavelength Exchange in a Highly-nonlinear Fiber" ECOC'01. We.L.1.3 (2001).

[3]. K. Uesaka. et.al., "Wavelength Exchange in a HNL-DSF -- Theory and Experiments" J. Sel. Top. Quant. Elect. 8, 560-568 (2002).

[4]. O. F. Yilmaz, et.al., "Time-slot Interchange of 40Gbs Variable Length Optical Packets Using Conversiondispersion-based Tunable Delays" Opt. Let. 33. 1954-1956 (2008).

[5]. D. J. Blumenthal, et.al., "All-Optical Lablel Swapping Networks and Technologies" J. Lightwave Technol. 18. 2058-2075(2000).

[6]. G. Kalogerakis. et.al., "Low Noise Figure Efficient Wavelength Exchange in an Optical Fiber" ECOC'06. 4800929 (2006).

[7]. K. Uesaka. et.al., "Polarization-insensitive wavelength exchange in HNL-DSF" OFC'02. ThY3(2002).

[8]. K. Y. Cheung, et.al,. "All Optical Packet Switching by Pulsed-Pump Wavelength Exchange in a HNL-DSF" OFC'07. OTUB4 (2007). 